\title{
Hommage
}

\section{Hélène Chaigneau (1919-2010)}

A tribute to

Hélène Chaigneau M.D.

*Psychiatre des hôpitaux, ancien interne et assistant du CTRS de Ville-Evrard, ancien membre du comité de rédaction de Perspectives Psy, 7, place Pinel, 75013 Paris, France. jean.garrabe@ wanadoo.fr

\author{
Jean GARRABÉ*
}

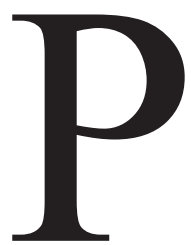

aradoxalement, le chef de service qui a sans doute initié le plus grand nombre d'internes des Hôpitaux psychiatriques de la Seine aux subtilités de ce mouvement théorico-pratique que Georges Daumézon et Philippe Koechlin ont, en 1952, dénommé psychothérapie institutionnelle, le Dr Hélène Chaigneau dont nous avons déploré la disparition le 24 août dernier, n'avait pas elle-même effectué son internat dans ces établissements. En effet, née dans une famille originaire de la région de Grenoble, elle a fait ses études de médecine dans l'École de cette ville, puis à la Faculté de Lyon. Elle n'était pas en ce temps là attirée par cette étrange spécialité médicale qu'est la psychiatrie et son premier contact avec elle, alors qu'elle accompagnait un de ses maîtres cardiologue à l'Hôpital Saint-Egrève pour surveiller les effets sur le cœur de malades mentaux traités par des chocs au cardiazol, ne lui avait pas donné une opinion très favorable sur les thérapeutiques alors pratiquées dans ces institutions ; elle comptait plus sur celles mises en œuvre dans des services comme celui de Paul-F. Girard (1882-1974) à l'Hôpital Neurologique de Lyon où elle a poursuivi ses études. Venue à Paris pour connaître l'enseignement d'Henri Ey (1900-1977), elle fut mise en contact par l'intermédiaire de Sven Follin (1911-1997) avec Paul Sivadon (1907-1992). Celui-ci lui offrit un poste de médecin assistant au Centre de Traitement et de Réadaptation Sociale qu'il dirigeait à l'hôpital de VilleEvrard, un des quatre CTRS créés après la Libération, avec ceux de Villejuif, Bonneval et Saint-Alban. Hélène Chaigneau présenta avec Paul Sivadon et Philippe Koechlin, luimême disparu il y a quelques mois, et qui était alors interne dans le service de Georges Daumézon à Maison-Blanche, une communication "Personnalisation et latéralisation 》 à la session de 1951 du Congrès des aliénistes et neurologistes de France et des pays de langue française à Rennes. Elle a en 1953 participé aux Entretiens psychiatriques organisés par Henri Ey à Sainte-Anne pour concourir au Prix que L'Évolution Psychiatrique réservait aux jeunes collègues en faisant une conférence « Réflexions sur la gaucherie et l'ambimanie "; bien qu'elle n'ait pas gagné ce prix, notre maître fit l'éloge de son goût pour la recherche et son esprit clinique.

Dès 1957, Hélène Chaigneau publia avec Philippe Koechlin dans L'Évolution Psychiatrique un article sur "Le champ asilaire. Études préliminaires à une psychothérapie de la situation carcérale » qui annonçait le thème de leurs recherches ultérieures.

La fondation par Paul Sivadon et plusieurs de ses collaborateurs du CTRS, dont Hélène Chaigneau, de l'Association privée Loi de 1901 L'Élan retrouvé allait permettre d'ouvrir à Paris les premières structures extra-hospitalières, hôpitaux de jour ou foyer de post-cure, 
ce que la réglementation en vigueur ne permettait pas aux hôpitaux publics de faire. Hélène Chaigneau est restée très active tout au long de sa carrière et même après sa retraite à l'Élan, en particulier en ce qui concerne la formation du personnel de toutes catégories qui y travaille à la relation avec les personnes souffrant de troubles mentaux.

Reçue au concours du médicat des hôpitaux psychiatriques, Hélène Chaigneau dut, comme le prescrivait la règle instaurée par le Ministère pour pallier la pénurie de psychiatres dont souffrait notre pays, occuper d'abord des chefferies de service dans des hôpitaux psychiatriques de départements provinciaux. La qualification officielle du Conseil de l'Ordre était celle de neuro-psychiatre de sorte que nombre de ces médecins des hôpitaux psychiatriques ne l'avaient pas ; ce n'est que plus tard que les internes des hôpitaux psychiatriques de la Seine obtinrent une équivalence sous réserve de justifier un stage en neurologie, puis que la neuropsychiatrie fut scindée en deux spécialités distinctes, réformes dans lesquelles le GEPPSS fondé par le comité de l'internat des hôpitaux psychiatriques de la Seine a joué un grand rôle. Hélène Chaigneau occupa donc d'abord un poste à l'hôpital de Niort, puis à celui de Prémontré où un autre chef de service était Paul-Claude Racamier (1924-1996), qui tentait déjà alors de repenser le rôle de la psychanalyse dans la réalité de la pratique hospitalière publique de la psychiatrie.

$\mathrm{Au}$ bout de ces années d'exil provincial, Hélène Chaigneau, promue médecin des hôpitaux psychiatrique de la Seine, eut la chance d'être nommée médecin-chef du CTRS de Vile-Evrard car Sven Follin qui avait succédé à ce poste à Paul Sivadon, lui-même parti pour diriger les services psychiatriques de la Mutuelle Générale de l'Éducation mis en place à partir de l'Institut Marcel Rivière au Château de la Verrière, préféra occuper celui de médecin-chef d'une des Sections femmes de Sainte-Anne qui était devenu vacant. De sorte que personnellement, libéré des obligations militaires liées aux « événements d'Algérie » comme on disait, je fus un des premiers internes des hôpitaux psychiatriques de la Seine à choisir son service à Ville-Evrard. J'y revins d'ailleurs à la fin de mon internat quand, reçu cette fois au médicat des hôpitaux psychiatriques, je pris l'un de ces postes d'assistant que nous pouvions occuper pendant trois ans. Les six postes offerts au choix de l'internat étaient fort recherchés en raison de l'enseignement qu'ils permettaient de recevoir. Cet enseignement était essentiellement clinique, centré soit autour de la rédaction des certificats de la loi du 30 juin 1838, tâche dévolue aux assistants qui « avaient la signature » et s'efforçaient de justifier le placement sous le régime du service libre plus propice à leur réadaptation du plus grand nombre possible de malades, soit au cours d'examens conjoints avec « la patronne » pour des malades posant des questions sémiologiques ou diagnostiques difficiles, déterminant des indications thérapeutiques particulières. Il faut dire que le taux de « surencombrement» était couramment dans les services d'hommes de $120 \%$ ou $130 \%$ de la capacité hospitalière et que les structures extra-hospitalières se réduisaient aux consultations du dispensaire de l'Office Public d'Hygiène Sociale, rue Léon Giraud, dans le XIX ${ }^{\mathrm{e}}$ arrondissement, d'où procédaient les malades internés. La mise en place de la politique de secteur préconisée par la circulaire du 15 mars 1960 paraissait encore un objectif lointain.

Hélène Chaigneau et ses collaborateurs parvenions à participer à de grandes réunions internationales. Ainsi, en 1966, nous nous fûmes autorisés par la Préfecture à nous absenter tous les deux, laissant le service sous la garde d'un autre assistant, René Girard, pour faire partie de la délégation française au VI ${ }^{\mathrm{e}}$ Congrès Mondial de psychiatrie organisé à Madrid par le Pr Juan-José Lopez Ibor (1906-1991). Nous eûmes de nombreuses surprises : la venue, grâce à Léon Chertok (1901-1991), en Espagne franquiste d'une délégation officielle de l'Association des psychiatres soviétiques avec discussion de la notion de " schizophrénie torpide » introduite par Snezhnevsky, la présentation d'un ordinateur IBM permettant de faire un diagnostic de Trouble Mental en cochant des items sur une fiche perforée sans avoir à " dialoguer avec l'insensé », etc. Mais nous y retrouvâmes aussi nos maîtres, Monsieur Minkowski (1885-1972) qui assistait là à son dernier grand congrès international, Paul Sivadon et Henri Ey qui allait abandonner à l'issue de ce congrès les fonctions de secrétaire général de l'Association Mondiale de Psychiatrie qu'il a occupé pendant seize ans, depuis de Premier Congrès Mondial de Paris, et qui, à cette occasion, prononça une conférence « La dissolution de la conscience dans le sommeil et le rêve et ses rapports avec la psychopathologie » qui constitue son testament scientifique.

Je me demande pourquoi, lorsque le Groupe d'Études de psychiatrie et neurologie fondé par le Comité de l'internat des hôpitaux psychiatriques de la Seine lança sa revue 
Perspectives Psychiatriques, nous ne lui avons pas demandé de faire partie de son comité de parrainage alors que plusieurs des membres du comité de rédaction avions été ses internes.

En revanche, lorsqu'en 1968 parurent deux nouvelles revues aux orientations très différentes pour ne pas dire opposées, elles demandèrent toutes deux au Dr Hélène Chaigneau de faire partie de leur comité de rédaction : la première, Confrontations psychiatriques, avait été fondée avec l'aide de Specia ; la seconde, Psychothérapie institutionnelle, était l'organe d'une Société de psychothérapie institutionnelle, héritière d'un groupe de réflexion, le $G$, qui ne publia qu'une demi-douzaine de numéros. Dans le $\mathrm{n}^{\circ} 2$ de Confrontations psychiatriques consacré à "l'évolution de la schizophrénie », elle publie "Prise en charge des sujets réputés schizophrènes " et, dans le $\mathrm{n}^{\circ} 1$ de Psychothérapie institutionnelle, "Réflexion à partir de la terminologie et du transfert dans l'institution », analysant à partir de son expérience dans le service public les obstacles qui se dressent dans le traitement de tels sujets. Soulignons que la majorité des membres de cette Société de Psychothérapie institutionnelle se réclamait du marxisme et du lacanisme et souvent des deux en même temps. Le catholicisme pratiquant d'Hélène Chaigneau faisait tache, d'autant qu'elle eut l'idée singulière, alors qu'elle occupait déjà un rang élevé dans la hiérarchie hospitalière, d'entreprendre, comme internes et assistants plus jeunes le faisions, un analyse personnelle et de choisir pour ce faire un didacticien, Sacha Nacht (1901-1977), fondateur de l'Institut de Psychanalyse de Paris dont l'opposition à Jacques Lacan dans ces années 1962-1964 était notoire (Paul Racamier était, lui, proche de Nacht et a présidé cet Institut). $\mathrm{Ce}$ catholicisme valait à Hélène Chaigneau d'être considérée comme une autorité en santé mentale dans des milieux religieux auxquels n'avaient pas accès d'autres psychiatres, comme les ordres cloîtrés féminins, et d'être consultée à ce propos et de conseiller des aumôniers des hôpitaux psychiatriques dans leur relations avec les malades. Il faut remarquer qu'un grand nombre des promoteurs de la psychothérapie institutionnelle étaient fils de pasteurs, pour lesquels le seul moyen de ne pas être obligés de succéder à leur père dans cette fonction, était de devenir psychiatre, ce qui reviendrait un peu au même si j'en crois ce qu'en a dit notre maitre Paul Sivadon.

Hélène Chaigneau et Pierre Chanoit, ayant été chargés de présenter un rapport sur les Thérapies institutionnelles lors de la LXIX ${ }^{\mathrm{e}}$ session du Congrès de Psychiatrie et de Neurologie de Langue Française qui devait se tenir à Caen, me firent l'amitié de m'associer à sa rédaction. Les échanges réguliers que nous eûmes pendant des mois pour ce faire furent particulièrement enrichissants. La présentation et la discussion de ce rapport se fit dans une ambiance assez surréaliste : en effet, cette session du CPNLF était présidée par notre ami Gaston Ferdière (1907-1990), qui fut violemment interpellé par ses anciens amis présents dans la salle venus lui reprocher les électrochocs faits à Antonin Artaud (1996-1948) qu'il avait accueilli à leur demande à Rodez en zone libre pour lui éviter de mourir de faim à Ville-Evrard. Je ne peux m'empêcher de penser à ces malades anonymes traités par choc au cardiazol à Saint-Egrève dont Hélène Chaigneau a évoqué le souvenir. Notre rapport fut longtemps considéré comme l'ouvrage de référence sur le sujet. Nous y faisions remarquer que tous les travaux sur la psychothérapie institutionnelle avaient jusque-là porté sur des institutions isolées et closes comme l'étaient les hôpitaux psychiatriques, héritiers des asiles d'aliénés, mais que leur ouverture et le développement de structures extra-hospitalières et des formes d'hospitalisation à temps partiel nécessitait une réflexion nouvelle qui avait d'ailleurs été initiée à L'Élan retrouvé.

De fait, la mise en place de la politique de secteur préconisée par la circulaire du 15 Mars 1960 et le nouveau découpage départemental de la région Île-de-France allait entraîner un tournant dans la carrière du Dr Chaigneau. En effet, des deux hôpitaux psychiatriques de la Seine sis à Neuilly-sur-Marne, l'un, celui de Ville-Evrard, fut dévolu au nouveau département de la Seine-Saint-Denis (93) et l'autre, Maison-Blanche, à celui de Paris (75) ; en outre, était instaurée la mixité tant des malades que du personnel dans les services sectorisés. Ceci amena Hélène Chaigneau - pour continuer à suivre les malades du $\mathrm{XIX}^{\mathrm{e}}$ arrondissement qu'elle avait en charge - à passer avec un petit nombre de ses collaborateurs à Maison Blanche pour y prendre en 1972 la chefferie d'un service sectorisé mixte. Cette mixité suscita des craintes de la part des infirmières confrontées à la violence physique des malades hommes. Par ailleurs, la mise en place des structures extra-hospitalières se fit d'une manière très économico-administrative avec, contrairement à ce que nous avions vécu à Ville-Evrard, des difficultés de communication entre la Direction et les chefs de secteurs. Ceci n'empêcha pas le service d'Hélène Chaigneau à Maison Blanche de continuer 
à être recherché par les internes jusqu'à son départ en retraite en 1987. Ce sont les membres de l'équipe du $26^{\mathrm{e}}$ secteur qui ont publié dans Le Monde le faire part qui a appris son décès à beaucoup de ses élèves.

Son départ en retraite n'avait pas diminué son activité dans d'autres lieux de transmission que l'hôpital. Personnellement, j'ai continué à la rencontrer aux réunions de L'Évolution psychiatrique, de L'élan, de Confrontations psychiatriques auxquelles elle était restée très assidue tant que son état de santé le lui permit. Mais je sais qu'elle a constamment été invitée à intervenir dans des institutions dirigées par ses anciens élèves tant en France que dans d'autres pays comme la Grèce ; le texte de ses interventions n'a pas toujours été publié mais, ces derniers mois, a été entreprise leur recollection pour faire figurer une bibliographie aussi complète que possible de ses travaux dans des recueils en préparation dont elle espérait qu'ils seraient publiés avant sa mort. Je lis dans "Sur la transmission en psychiatrie », texte qu'elle a écrit en hommage à Sivadon: "S'il doit exister une différence entre un grand fou et un grand psychiatre, elle réside peut-être en ce que le grand fou s'est bien trop approché de la folie alors que le grand psychiatre ne s'est jamais trop approché des fous. Mais il n'en transmet que ce $q u$ 'il en peut raisonnablement penser $》$.

Je me permets de la lui emprunter pour rendre à mon tour hommage à Hélène Chaigneau qui fut un grand psychiatre.

\begin{tabular}{|c|c|}
\hline \multirow[t]{3}{*}{ Communiqué } & \\
\hline & $\begin{array}{c}\text { Institut Mutualiste Montsouris } \\
\text { Département de Psychiatrie de l'Adolescent } \\
\text { Direction et coordination : Pr M. Corcos \& C. Dugré-Le Bigre } \\
42 \text {, Boulevard Jourdan-75014 Paris } \\
\text { Amphithéâtre de l'IMM, Hall d'accueil } \\
\text { M. Porte d'Orléans - RER Cité Universitaire } \\
\text { E : } 01.56 .61 .69 .19 \text { - Contact : corinne.dugre-lebigre@imm.fr }\end{array}$ \\
\hline & $\begin{array}{l}\text { Programme } 2011 \\
\text { SÉMTNATRE BABYLONE } \\
\text { PSYCHANALYSE, LITTERATURE ET ART }\end{array}$ \\
\hline $\begin{array}{l}\Rightarrow \text { Lundi } 4 \text { janvier } 2011 \\
\text { à } 20 \mathrm{~h} 30 \\
\text { LITTERATURE }\end{array}$ & $\begin{array}{l}\text { Yoann LOISEL } \\
\text { A propos de film mis en scène par Samuel Beckett, ou : } \\
\text { Comment Buster Keaton s'est retrouvé sur le chemin de Samuel Beckett } \\
\text { Discutant : Denis BOCHEREAU }\end{array}$ \\
\hline $\begin{array}{l}\Rightarrow \text { Lundi ler février } 2011 \\
\text { à } 20 \mathrm{~h} 30 \\
\text { LITTERATURE }\end{array}$ & $\begin{array}{l}\text { Isée BERNATEAU } \\
\text { Proust et la séparation } \\
\text { Discutant : Maurice CORCOS }\end{array}$ \\
\hline $\begin{array}{l}\Rightarrow \text { Lundi } 7 \text { mars } 2011 \\
\text { à } 20 \mathrm{~h} 30 \\
\text { LITTERATURE } \\
\end{array}$ & $\begin{array}{l}\text { Paul DENIS } \\
\text { Paul Valéry et le divan du poème } \\
\text { Discutant: Elisabeth BIROT }\end{array}$ \\
\hline \multicolumn{2}{|c|}{$\begin{array}{c}\text { Vendredi } 25 \text { mars } 2011 \\
\text { IV ème COLLOQUE BABYLONE - Institut Mutualiste Montsouris } \\
\text { Journée organisée par les Séminaires BABYLONE et la Revue ADOLESCENCE } \\
\text { "Vérité et illusions à l'adolescence : terreurs et esquisses sauvages » } \\
\text { Intervenants : Maurice Corcos, Philippe Gutton, Silke Schauder, Alejandro Rojas-Urrego }\end{array}$} \\
\hline $\begin{array}{l}\Rightarrow \text { Lundi } 2 \text { mai } 2011 \\
\text { à } 20 \mathrm{~h} 30 \\
\text { LITTERATURE }\end{array}$ & $\begin{array}{l}\text { René KAES } \\
\text { Utopie rêveuse et utopie systématique. Espace transitionnel et paradoxe } \\
\text { dans l'Utopia de Thomas More } \\
\text { Discutant: Anne-Marie SMITH }\end{array}$ \\
\hline $\begin{array}{l}\Rightarrow \text { Lundi } 6 \text { juin } 2011 \\
\text { à } 20 \mathrm{~h} 30 \\
\text { LITTERATURE }\end{array}$ & $\begin{array}{l}\text { J.-D. NASIO } \\
\text { L'enfant merveilleux de la Callas, c'est sa voix } \\
\text { Discutant: Silke SCHAUDER }\end{array}$ \\
\hline
\end{tabular}

